\title{
Quantitative Assessment of Brain Tumor Radiation Treatment Reveals Decrease in Tumor-supporting Vessels
}

\author{
David Haberthür $^{1^{*}}$, Ruslan Hlushchuk ${ }^{1}$, Marine Potez ${ }^{1}$, Audrey Bouchet ${ }^{1}$ and Valentin Djonov ${ }^{1}$ \\ 1. Institute of Anatomy, University of Bern, Switzerland \\ * Corresponding author, haberthuer@ana.unibe.ch
}

Angiogenesis - the formation of new blood vessels - is an important factor for tumor growth (Sherwood, Parris, and Folkman 1971). Reducing tumor-supporting vessels by radiation treatment is a powerful option for treating tumors; specialized treatments that deliver high radiation doses have shown to enable excellent survival rate (J. A. Laissue et al. 1998). Among rodent models for brain tumors, the 9L-gliosarcoma model is a widely used one, since it mimics important features of human brain tumor growth. In this study we extracted and assessed biologically relevant values of brain tumors (tumor and vessel volume, vessel surface, etc.) from rats that underwent different radiation treatments. These values are extracted based on two imaging modalities, namely microCT and magnetic resonance imaging (MRI).

Brain tumors were induced in 10-week-old Fisher 344 rats $(n=59)$ by inoculation of gliosarcoma cells into the right caudate nucleus through the skull (Bouchet et al. 2014). Ten days after inoculation, tumor volume was assessed by MRI performed with a 4.7 T Bruker Avance III console (Lemasson et al. 2015). Based on tumor volume, animals were split into groups with comparable tumor size ready for either microbeam radiation therapy (MRT), conventional radiation therapy (so called broad beam, BB) or no therapy (CTRL).

Irradiation was performed at the ID17 biomedical beamline at the European Synchrotron Radiation Facility. Rats were irradiated ten days after inoculation using two 8 x $10 \mathrm{~mm}$ irradiation fields focused onto the tumor location in the anterior part of the right hemisphere. For the MRT-animals, the irradiation field was split into 40 microbeams (width $50 \mu \mathrm{m}, 200 \mu \mathrm{m}$ on-center spacing) using a multislit collimator. The in-microbeam entrance dose was $250 \mathrm{~Gy}$, the valley dose approximately 9 Gy. For the BB-animals, the irradiation was homogeneously applied to the same area with a dose equivalent to the MRT valley dose.

On days 6, 10 and 14 after radiation treatment, rats were again imaged by MRI. Subsequently, they were infused with a contrast agent ( $\mu$ Angiofil, Schaad et al. (2017)) and their brains extracted. Fifty-four of those brains were imaged with a Bruker SkyScan 1272 at $5 \mu \mathrm{m}$ voxel size.

After manually delineating the tumor regions of interest (ROIs) in CTAn we used a custom image processing pipeline in Python to assess the data sets and extract the aforementioned values for 54 tumors.

Tumor ROIs can easily be visualized. Figure 1 shows three example tumors from the whole set of scans. Radiation treatment reduces the vasculature in the tumor, as can be seen in Figure 2. Using the described approach, we show that radiation treatment decreases the vasculature in the tumor, i.e. less vessels are available to provide the tumor with nutrients. Our unbiased, automatic assessment shows that the performed radiation treatment is successful. 

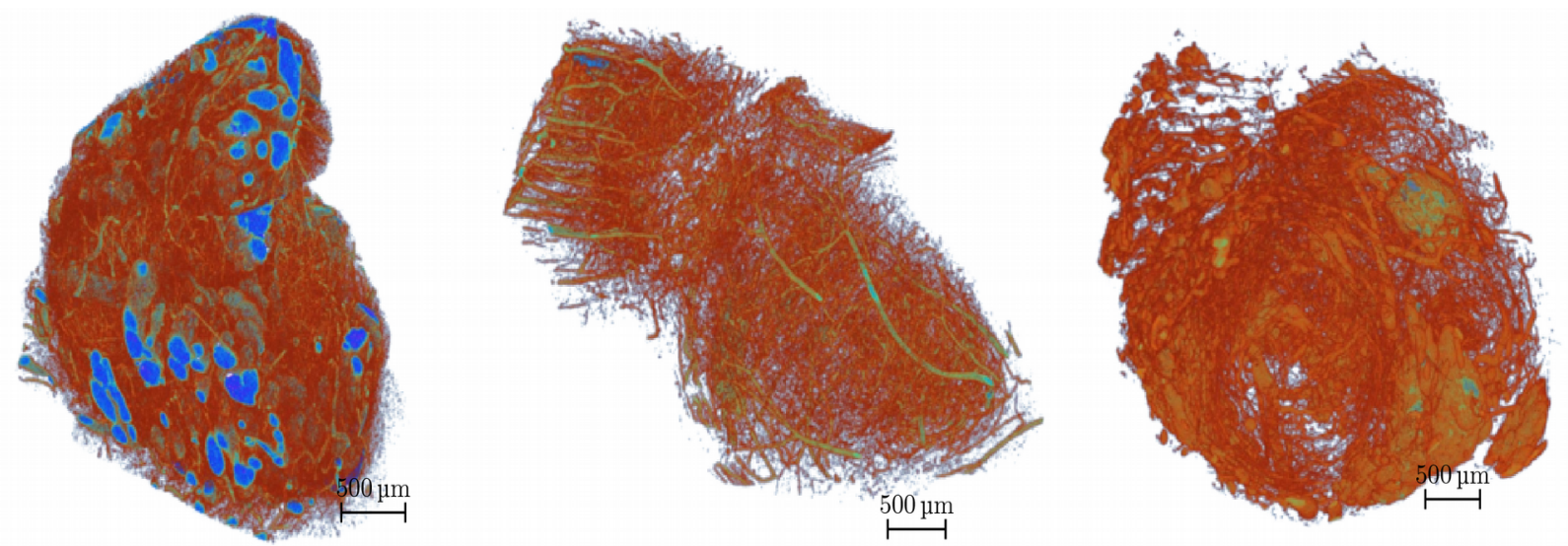

Figure 1. Visualization of three of the 54 scanned tumors. Left to right: Control, microbeam and broad beam radiation therapy.
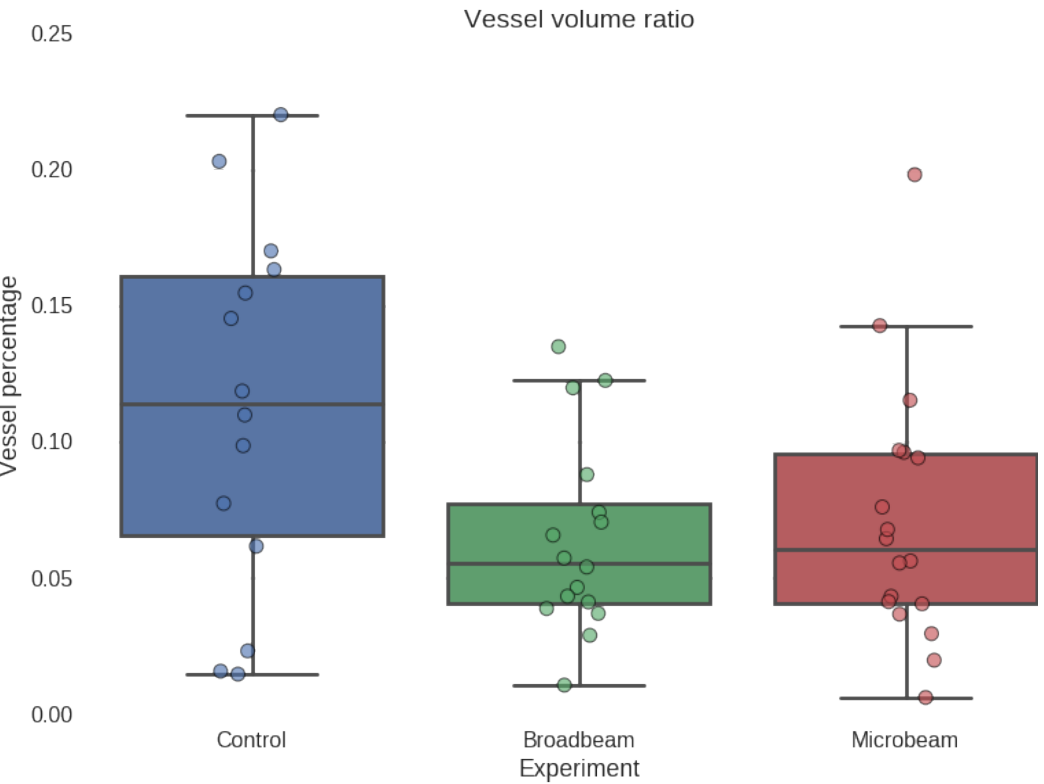

Figure 2. Box plots of the vessel volume per tumor volume.

References:

[1] A Bouchet et al., Characterization of the 9L gliosarcoma implanted in the Fischer rat: an orthotopic model for a grade IV brain tumor. Tumor Biology, (2014), doi:10.1007/s13277-014-1783-6

[2] JA Laissue, et al., Neuropathology of ablation of rat gliosarcomas and contiguous brain tissues using a microplanar beam of synchrotron-wiggler-generated X rays., International Journal of Cancer (1998), doi:10.1002/(SICI)1097-0215(19981123)78:5<654::AID-IJC21>3.0.CO;2-L

[3] B Lemasson et al., Multiparametric MRI as an early biomarker of individual therapy effects during concomitant treatment of brain tumours., NMR in Biomedicine (2015), doi:10.1002/nbm.3357

[4] L Schaad et al., Correlative Imaging of the Murine Hind Limb Vasculature and Muscle Tissue by MicroCT and Light Microscopy., Scientific Reports (2017), doi:10.1038/srep41842

[5] LM Sherwood et al., Tumor Angiogenesis: Therapeutic Implications., New England Journal of Medicine (1971), doi:10.1056/NEJM197111182852108 